\title{
Numerical Study of Focusing Parameters of a Five-Electrode Cathode Lens with the Rotational Symmetry of the Field
}

\author{
Ibraev A.T. ${ }^{1, a}$, Ibraev A.A. ${ }^{2, b}$, Kuttybayeva A.E. ${ }^{1, c}$, Sagyndyk $A^{1, d}$, Junussova \\ $\mathrm{D}^{1, \mathrm{e}}$
}

${ }^{1}$ Department of radio engineering, electronics and telecommunications Satpayev, Kazakh National Technical University, Kazakhstan

${ }^{2}$ Kazakhstan Academy of Information and Business, Kazakhstan.

Keywords: cathode lens, focalization, electron, ion, instrument, aberration.

Abstract. The paper presents some results of numerical studies and analyzes of paraxial parameters and spatial aberrations of a five-electrode cathode lens with rotationally symmetric field.

\section{Introduction}

Cathode, or emission lens, is one of the key elements of ion sources, charged particle accelerators, electronic optical instruments and ion-beam processing units in nano- and microelectronics, ion rocket engines and a number of analytical instruments and devices. Technical specifications of the mentioned above devices and analytical instruments directly depend on the cathode lens focalization quality.

The paper provides the numerical study and the analysis of paraxial parameters and spatial aberrations of a five-electrode cathode lens, which consists of a planar cathode and four successively and coaxially arranged electrodes of cylindrical shape with equal values of their diameters.

Let us introduce an $r, z, \psi$ cylindrical coordinate system. The cathode in this coordinate system is perpendicular to the $z$ main optical axis, and the reading of the coordinate values is taken from the cathode surface. The generatrices of the electrodes of a cylindrical shape (axisymmetric bodies) are parallel to the $z$ axis. The ccathode potential is zero, the potentials of the first, second, third and fourth cylindrical electrodes are denoted $\varphi_{1}, \varphi_{2}, \varphi_{3}$ and $\varphi_{4}$ respectively.

Due to the electrodes rotational symmetry the parameters of the lens under investigation do not depend on the $\psi$ coordinate.

\section{Discussed problems}

The focalization model of a five-electrode cathode lens with the rotational symmetry of the field

The distribution of the electrostatic potential along the $\Phi(z)$ main optical axis of the lens can be sufficiently accurately calculated by the formula [1]:

$$
\begin{aligned}
& \Phi(z)=\varphi_{1} \operatorname{th}\left(1.318 \frac{z}{R}\right)+ \frac{1}{2}\left(\varphi_{2}-\varphi_{1}\right)\left[\operatorname{th}\left(1.318 \frac{z+z_{1}}{R}\right)+\operatorname{th}\left(1.318 \frac{z-z_{1}}{R}\right)\right]+ \\
&+\frac{1}{2}\left(\varphi_{3}-\varphi_{2}\right)\left[\operatorname{th}\left(1.318 \frac{z+z_{2}}{R}\right)+\operatorname{th}\left(1.318 \frac{z-z_{2}}{R}\right)\right]+ \\
&+\frac{1}{2}\left(\varphi_{4}-\varphi_{3}\right)\left[\operatorname{th}\left(1.318 \frac{z+z_{3}}{R}\right)+\operatorname{th}\left(1.318 \frac{z-z_{3}}{R}\right)\right],
\end{aligned}
$$

where $z_{1}, z_{2}, z_{3}$ are the coordinates of the electrode ends remote from the cathode (by the gaps), of respectively, the first, second and third electrodes of cylindrical shape; $R$ is the radius of the electrodes of the cylindrical shape. The dimensions of the gaps between the electrodes are considered to be small magnitudes which can be neglected. 
In the paraxial approximation the motion of charged particles in the lens under study satisfies the equation $[2,3]$

$$
\Phi r^{\prime \prime}+\frac{1}{2} \Phi^{\prime} r^{\prime}+\frac{1}{4} \Phi^{\prime \prime} r=0
$$

The general solution of equation (2) has the form

$$
r(z)=r_{k} u(z)+\frac{2}{\Phi_{k}^{\prime}} \sqrt{\varepsilon_{r}} v(z) e^{i \beta},
$$

where $r_{k}$ is the coordinate of the emission point of a charged particle from the cathode; $\Phi_{k}^{\prime}$ is the value of the electrostatic field intensity at the cathode; $\varepsilon_{r}$ - the radial component of the initial energy of the particles emitted from the cathode; $\beta$ is the angle between the planes, one of which passes through the $z$ axis and the point of particle emission, the other goes through the initial velocity vector and the line parallel to the $z$ axis which starts from the point of the particle emission; $u(z)$ and $v(z)$ are particular linearly independent solutions of the paraxial equation (2).

The solution $u(z)$ satisfies the equation (2) with the initial conditions

$$
u(0)=1, \quad u^{\prime}(0)=0 .
$$

Due to the peculiarity in equation (2) at $z=0$ the second particular linearly independent solution $v(z)$ is determined from the expression

$$
v(z)=\sqrt{\Phi(z)} w(z)
$$

where $w(z)$ is the solution of the equation

$$
\Phi w^{\prime \prime}+\frac{3}{2} \Phi^{\prime} w^{\prime}+\frac{3}{4} \Phi^{\prime \prime} w=0
$$

with the following initial conditions

$$
w(0)=1, \quad w^{\prime}(0)=0 .
$$

The calculation of the cathode lens parameters was made on condition it forms a crossover, i.e. on condition that

$$
\left.u(z)\right|_{z=z_{c}}=0 .
$$

At this time $z_{c}$ denotes the coordinate of the charged particle beam crossover formed by the lens under consideration.

Following computer-aided numerical calculations we defined the lens parameter ratios (electrode sizes, electrode potentials) at which the condition (8) is fulfilled. We assumed $\varphi_{1}=\varphi_{3}$ and $\varphi_{2}=\varphi_{4}=1$, $z_{1}=z_{2}-z_{1}=z_{3}-z_{2}$. The results are presented in the form of the diagram (Fig.1). At carrying out calculations we accepted $R=1$. In the following diagrams Line 1 (with square dots) corresponds to the value $z_{c}=5 R$, Line 2 (triangles) corresponds to $z_{c}=10 R$, Line 3 (X dots) corresponds to $z_{c}=15 R$, Line 4 (G dots) - to $z_{c}=20 R$.

The coordinate of a random particle with nonzero initial energy in the crossover plane can be defined by the formula

$$
r\left(z_{c}\right)=\frac{2}{\Phi_{k}^{\prime}} \sqrt{\varepsilon_{r}} v\left(z_{c}\right) e^{i \beta}
$$

Based on the expression (9) it can be seen that the size of the beam crossover in paraxial approximation for various values of $z_{c}$ depends on the values of $\Phi_{k}^{\prime}$ and $v\left(z_{c}\right)$. 
$z_{1}$

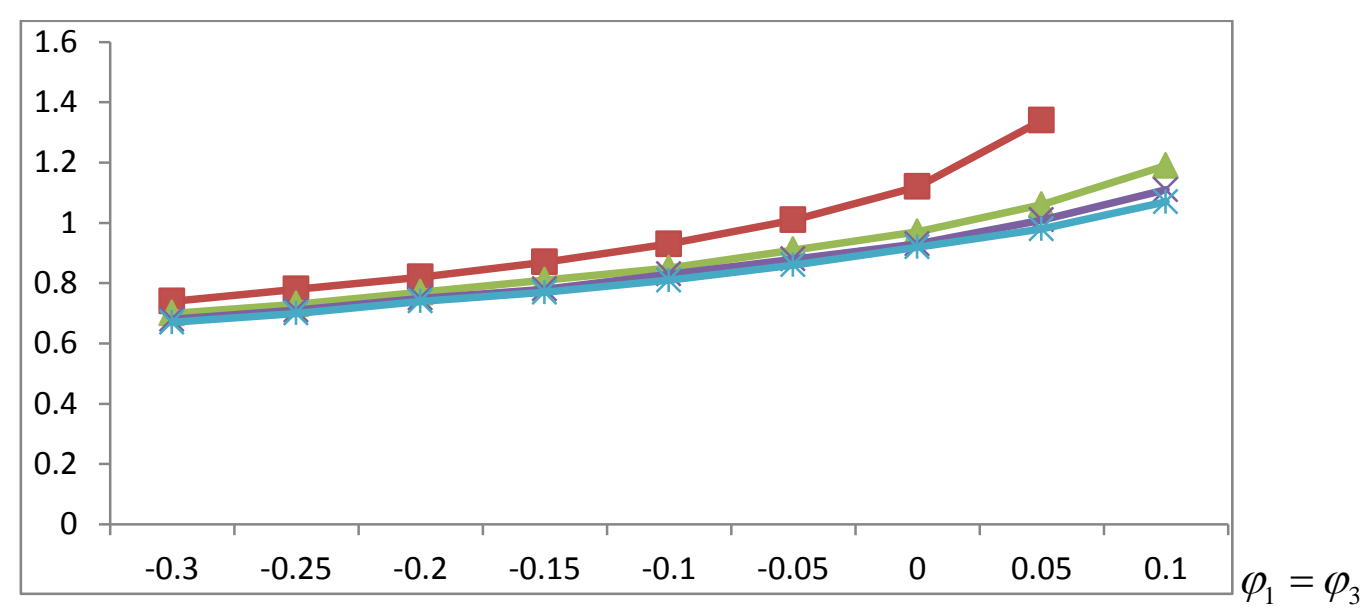

Fig. 1: The crossover formation conditions

Individual aberrations of the lens under study can be identified by analyzing the equation of the trajectories of charged particles [2,3]

$$
\begin{aligned}
r e^{i(\psi+\theta)}=r_{k} u(z)+ & b_{1} v(z)+r_{k} \sqrt{\varepsilon_{z}} C_{21}(z)+b_{1} \sqrt{\varepsilon_{z}} C_{22}(z)+ \\
+r_{k}^{3} C_{31}(z)+r_{k}^{2} & \bar{b}_{1} C_{32}(z)+r_{k}^{2} b_{1} C_{33}(z)+r_{k} b_{1}^{2} C_{34}(z)+ \\
& +r_{k} b_{1} \bar{b}_{1} C_{35}(z)+b_{1}^{2} \bar{b}_{1} C_{36}(z)+r_{k} \varepsilon_{z} C_{37}(z)+b_{1} \varepsilon_{z} C_{38}(z)
\end{aligned}
$$

Aberrational coefficients $C_{n j}(n=2,3 ; j=1,2, \ldots, 8)$ are in the form [4]

$$
\begin{aligned}
& C_{21}=B_{21}-u^{\prime} \zeta_{1}, C_{22}=B_{22}-v^{\prime} \zeta_{1}, C_{31}=B_{31}-u^{\prime} \zeta_{21}, \\
& C_{32}=B_{32}-u^{\prime} \zeta_{22}, C_{33}=B_{33}-u^{\prime} \zeta_{22}-v^{\prime} \zeta_{21}, \\
& C_{34}=B_{34}-v^{\prime} \zeta_{22}, C_{35}=B_{35}-u^{\prime} \zeta_{23}-v^{\prime} \zeta_{22}, \\
& C_{36}=B_{36}-v^{\prime} \zeta_{23}, C_{37}=B_{37}-u^{\prime} \zeta_{24}, C_{38}=B_{38}-v^{\prime} \zeta_{24} .
\end{aligned}
$$

Herein

$$
\mathrm{B}_{n j}=-\frac{2}{\Phi_{k}^{\prime}}\left(u \int_{0}^{z_{0}} \frac{S_{n j}}{\sqrt{\Phi}} v d z_{0}-v \int_{0}^{z_{0}} \frac{S_{n j}}{\sqrt{\Phi}} u d z_{0}\right)
$$

where

$$
\begin{aligned}
& S_{21}=\frac{\sqrt{\Phi}}{2 \Phi_{k}^{\prime}} \Phi^{\prime \prime \prime} u, \\
& S_{22}=\frac{\sqrt{\Phi}}{2 \Phi_{k}^{\prime}} \Phi^{\prime \prime \prime} v \\
& S_{31}=\frac{\Phi^{I V}}{32} u^{3}-\frac{\Phi^{\prime \prime \prime}}{4} u \zeta_{21}, \\
& S_{32}=\frac{\Phi^{I V}}{32} u^{2} v-\frac{\Phi^{\prime \prime \prime}}{4} \zeta_{22} u, \\
& S_{33}=\frac{\Phi^{I V}}{16} u^{2} v-\frac{\Phi^{\prime \prime \prime}}{4}\left[\zeta_{22} u+v \zeta_{21}\right], \\
& S_{34}=\frac{\Phi^{I V}}{32} u v^{2}-\frac{\Phi^{\prime \prime \prime}}{4} v \zeta_{22}, \\
& S_{35}=\frac{\Phi^{I V}}{16} u v^{2}-\frac{\Phi^{\prime \prime \prime}}{4}\left[u \zeta_{23}+v \zeta_{22}\right],
\end{aligned}
$$




$$
\begin{aligned}
& S_{36}=\frac{\Phi^{I V}}{32} v^{3}-\frac{\Phi^{\prime \prime \prime}}{4} \zeta_{23} v, \\
& S_{37}=-\frac{\Phi^{\prime \prime \prime}}{4} u \zeta_{24}-\frac{\Phi^{I V} \Phi u}{2 \Phi_{k}^{\prime 2}}, \\
& S_{38}=-\frac{\Phi^{\prime \prime \prime}}{4} v \zeta_{24}-\frac{\Phi^{I V} \Phi v}{2 \Phi_{k}^{\prime 2}} .
\end{aligned}
$$

The expressions $\zeta_{21}-\zeta_{24}$ the latter contain are defined by the formulae

$$
\begin{array}{r}
\zeta_{21}=\frac{1}{2 R}-\frac{u u^{\prime}}{2}+\sqrt{\Phi} \int_{0}^{z_{0}} \frac{u u^{\prime \prime}}{\sqrt{\Phi}} d z_{0}, \\
\zeta_{22}=\frac{\sqrt{\Phi}}{2} \int_{0}^{z_{0}}\left(u^{\prime \prime} v-u^{\prime} v^{\prime}\right) d z_{0}, \\
\zeta_{23}=\frac{\sqrt{\Phi}}{2} \int_{0}^{z_{0}} \frac{1}{\Phi \sqrt{\Phi}}\left(\frac{\Phi_{k}^{\prime 2}}{4}-\Phi v^{\prime 2}-\frac{\Phi^{\prime \prime}}{4} v^{2}\right) d z_{0}, \\
\zeta_{24}=\frac{\sqrt{\Phi}}{2} \int_{0}^{z_{0}} \frac{1}{\Phi \sqrt{\Phi}}\left[1+\frac{1}{\Phi_{k}^{\prime 2}}\left(2 \Phi \Phi^{\prime \prime}-\Phi^{\prime 2}\right)\right] d z_{0} .
\end{array}
$$

The results of calculation of a number of aberration coefficients are represented in the graphs (Figure 2 - Figure 9) which are provided below. These data makes clear that the values of aberrations are higher when the focusing electrode has a greater in absolute magnitude negative value of the applied potential, and the lens field is more curved $[5,6]$.

$C_{31}$

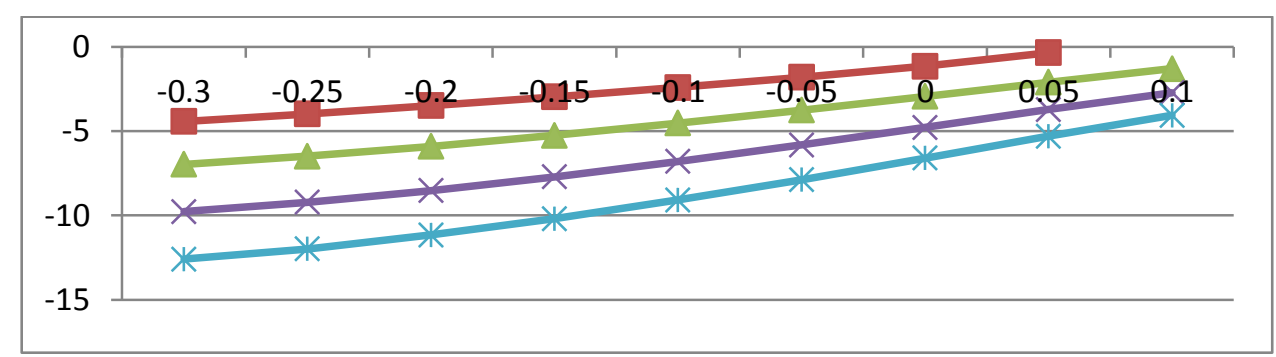

Fig. 2: $\mathrm{C}_{31}$ aberration coefficient graphs $\varphi_{1}=\varphi_{3}$

$C_{32}$

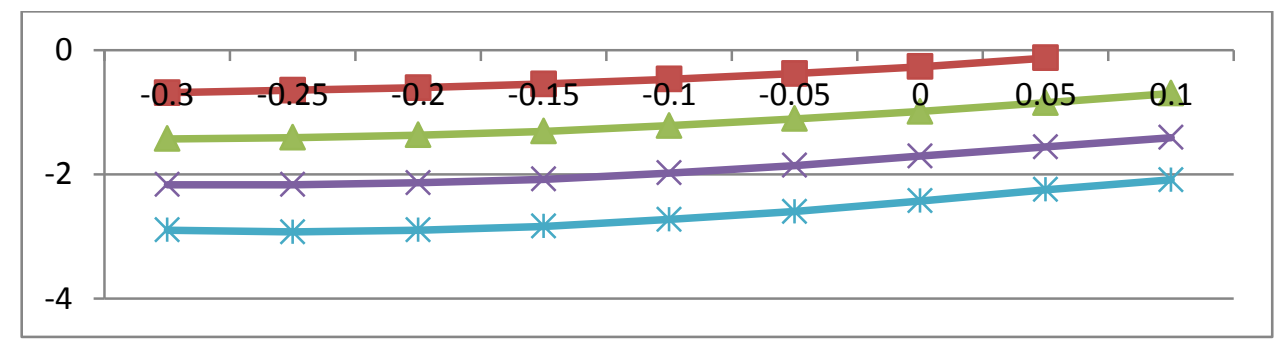

Fig.3: $\mathrm{C}_{32}$ aberration coefficient graphs $\varphi_{1}=\varphi_{3}$

$C_{33}$

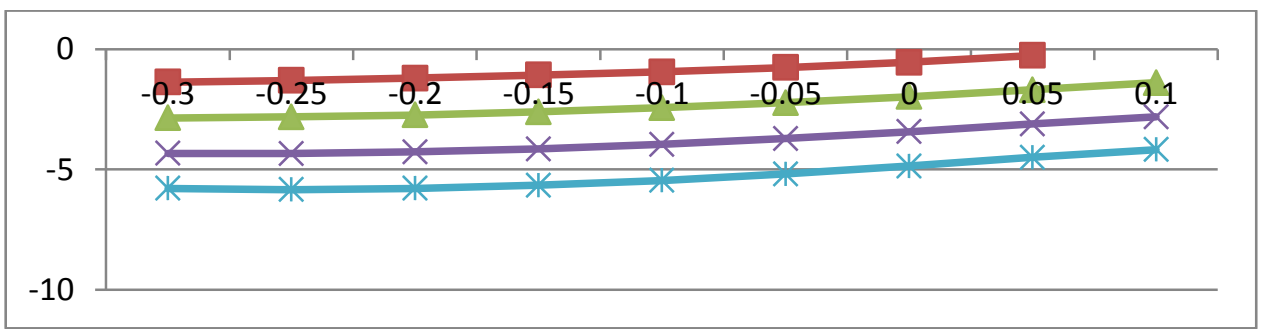

Fig. 4: $\mathrm{C}_{33}$ aberration coefficient graphs $\varphi_{1}=\varphi_{3}$ 
$C_{34}$

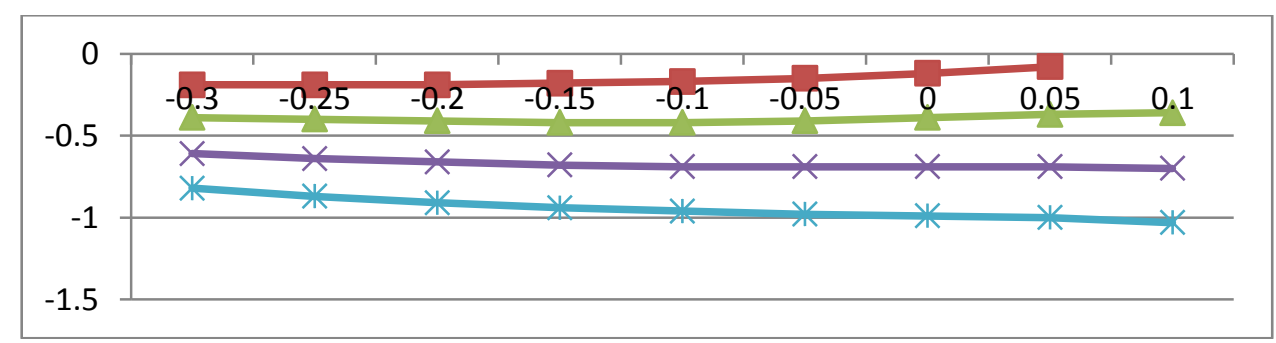

Fig.5: $\mathrm{C}_{34}$ aberration coefficient graphs $\varphi_{1}=\varphi_{3}$

$C_{35}$

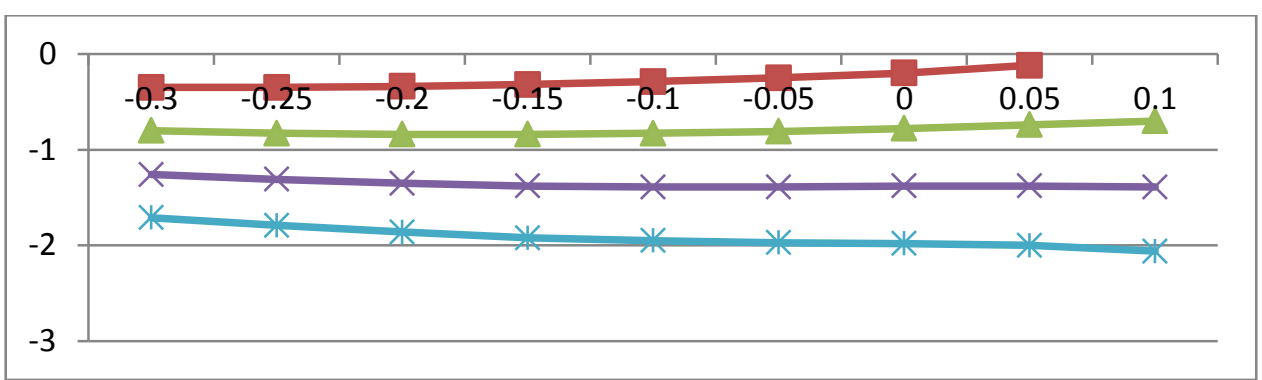

Fig.6: $\mathrm{C}_{35}$ aberration coefficient graphs $\varphi_{1}=\varphi_{3}$

$C_{36}$

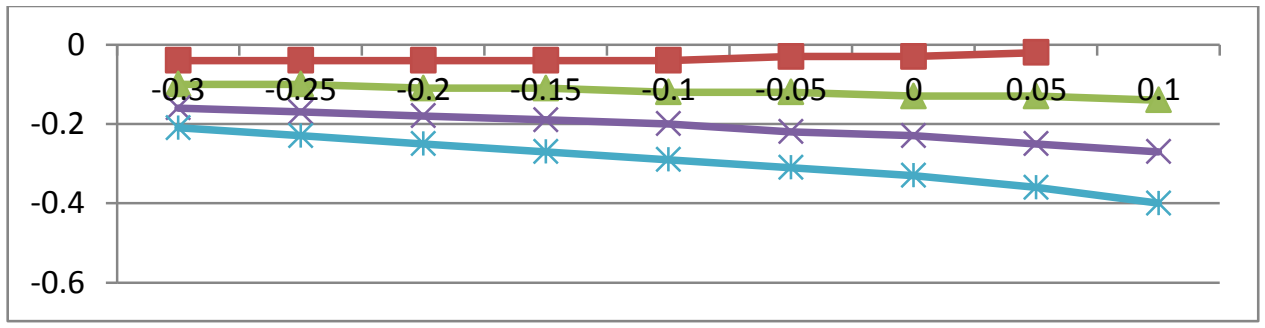

Fig.7: $\mathrm{C}_{36}$ aberration coefficient graphs $\varphi_{1}=\varphi_{3}$

$C_{37}$

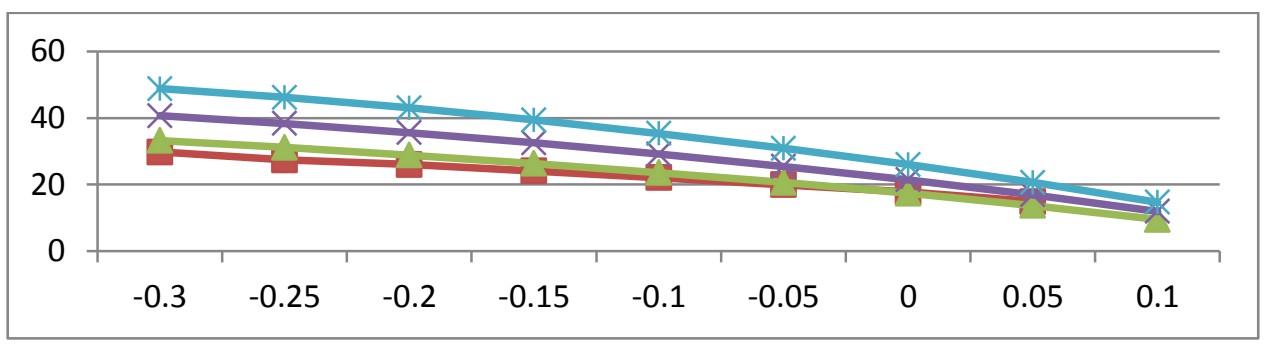

Fig. 8: $\mathrm{C}_{37}$ aberration coefficient graphs $\varphi_{1}=\varphi_{3}$

$C_{38}$

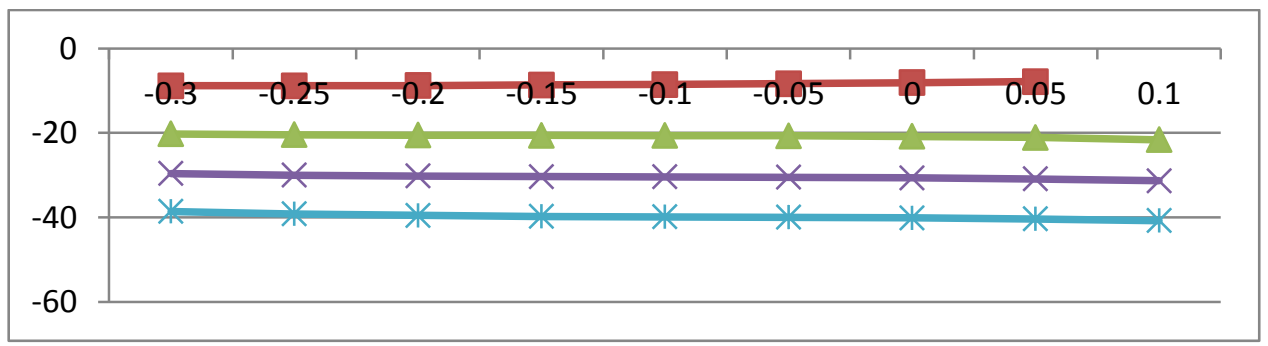

Fig.9: $\mathrm{C}_{38}$ aberration coefficient graphs $\varphi_{1}=\varphi_{3}$

\section{Conclusions}


The comparison of the obtained values of the aberrations of a five-electrode cathode lens with rotational symmetry with the results of numerical studies of 3-electrode axisymmetric and transaxial cathode lenses allows us to conclude that the increase in the number of electrodes lets reduce particular aberrations in comparison with 3-electrode rotationally symmetric lenses. However, transaxial lens in the horizontal direction allow obtaining considerably smaller values of aberrations similar in nature.

\section{References}

[1] Baranova L.A., Yavor S.J. The electrostatic electron lenses. - M:, Nauka, 1986, 190 p.

[2] Ibraev A.T. The study of the properties of an axisymmetric cathode lens. / Intercollegiate collection of research papers "Creating elements of the society information infrastructure." Almaty, 1996.

[3] Ibraev A.T. The theory of emission and wave-reflecting optical elements with the direct optic axis. - Prikladnaya Physica, M.: 2009. - No.3.- p. 106-110.

[4] Deutch S. and Deutch, A.: Understanding the Nervous System; An Engineering Perspective (IEEE Press, New York) 1993.

[5] Mandelbrot B.B.: The Fractal Geometry of Nature (Freeman, New York) 1983.

[6] Cohen J. and Stewart, I.: The Collapse of Chaos (Penguin, London) 1995. 